\title{
Geographic Information System to Monitor and Forecast the Influence of Foot-and-Mouth Disease of Poultry and Cattle
}

\author{
Le Vu Truong \\ Department of IT, FPT University, Ho Chi Minhcity, Vietnam \\ Huynh Tan Hoi \\ Department of Japanese, FPT University, Ho Chi Minhcity, Vietnam
}

\begin{abstract}
The paper introduces the origin, technical features, scale, application efficiency of geographic information system (GIS) to monitor the situation and forecast the spread of foot-and-mouth disease of poultry and cattle-a technological product of Vietnam Software Academy for Water. Application of the system will help the producers and managers to quickly capture the situation of raising poultry and cattle, vaccination results in the related territory, flu outbreaks, epidemic foot-and-mouth disease, and the spread of these two dangers as they arise, resulting in appropriate decisions and solutions in order to prevent, minimize the risk effectively.
\end{abstract}

Keywords: foot-and-mouth disease, GIS, poultry and cattle, solutions

\section{Introduction}

In recent years, the epidemic of cattle and poultry has become increasingly complex, causing serious harm to the economy, the living environment, and the health of the community. Especially, hidden dangerous risks are foot-and-mouth diseases. Disease causes direct loss to livestock farmers, imbalance of food supply, and demand in the market, affecting consumers. In addition, these foot-and-mouth diseases also adversely affect public health, the living environment and in some cases that lead to loss of lives, threaten the stability of socio-economy and pose the risk of becoming a human flu pandemic in the world.

It is worth mentioning that when the epidemic occurs, local and central management levels lack the information needed to assess the status and the potential danger to take right action to prevent in time. Apart from not having a complete system of investigation and information collection, we lack both storage and information processing facilities. Survey results from local authorities sent to FAX, parcel, or telephone are stored in the form of ballot papers, which makes the analysis and aggregation of information difficult, slow, and unable to meet the requirements of the management, monitoring the epidemic and make decisions to prevent. In other words, it takes information, takes time, effort, and energy to gather information, but lowers information efficiency. When drawing map of disease status, veterinarians must use a paper map and an old colouring method to demonstrate.

Le Vu Truong, M.A, Department of IT, FPT University, Ho Chi Minhcity, Vietnam.

Huynh Tan Hoi, PhD candiadate, Department of Japanese, FPT University, Ho Chi Minhcity, Vietnam.

Correspondence concerning this article should be addressed to Huynh Tan Hoi, Nam Hai Hotel, $79^{\text {tr }}$ Street, district 9, Ho Chi Minh City, Vietnam. Tel: 0084-965.460.459. 
Of course, by doing so, the map can't accurately reflect the actual situation. This is the consequence of the lack of a modern information system to store the types of information collected, to process them quickly and in a timely manner in order to give opinions to the administrators in many cases. In such a situation, the GIS and Remote Sensing Division of Vietnam Software Academy for Water have researched and developed a geographic information system that monitors the foot-and-mouth disease and forecasts the spread of the outbreak. The system was started in the framework of a provincial scientific research project of Vinh Phuc Province, completed and implemented in application in Nam Dinh Province and Nghe An Province within the framework of science and technology. In 2008, the Department of Agriculture and Rural Development together with Quang Ninh Department of Science and Technology raised the issue of upgrading and expanding the system to monitor and forecast the spread of both foot and mouth epidemic and apply it to the work of raising poultry and cattle of the province. Up to now, the GIS system has monitored the situation and predicted the spread of foot and mouth disease and poultry flu for the whole Quang Ninh Province. Through the article, a brief overview of the technical features of information systems will be presented, then their applicability, scope, and the effectiveness of application of the system to the monitoring and forecasting of infectious diseases of livestock are also introduced [2].

\section{Content of Writing Technical Features of the Information System}

\section{The Structure of the System}

The geographic information system monitors the situation and predicts the spread of foot-and-mouth disease of livestock. The host software includes a database, maps, and attribute data on the server, attributed management software, and spatial data. The client software is the client software - the user's tool to log into the system from their workstations, update the data into the system, exploit information from the system. If the server has an Internet address, the client software can log on to the system via the Internet. In case the server does not have an address on the Internet, the client software can connect to the server via a public telephone line or use VPN technology. The number of connectors at a time depends on the license to use attribute data management, spatial data management, and the ability of the connection port on the server [3].

\section{Database on Livestock Situation and Avian Flu, Foot and Mouth Disease}

As a GIS system, stored data include spatial data (maps) and attribute data. To be able to both serve the management of livestock situation, disease situation, both serve for forecasting, warning trend spread, map data as well as attribute data attached with detailed management to each clustered population, each farm. The following are the types of data stored in the system.

Map data:

+ Classes of administrative maps of communes, districts, and provinces;

+ Map of national, inter-provincial, inter-district, and inter-commune roads;

+ Grade of irrigation map: rivers, streams, ponds, and lakes;

+ Map of residential areas;

+ Map layers of poultry farms: chicken, bird, waterfowl;

+ Map of farms providing poultry breeds;

+ Classes of cattle farms: buffalos, cows, pigs, and goats;

+ Classes of farms providing livestock breeds; 
+ The map of the network of markets for cattle and poultry products;

+ Map of shops selling industrial feeds for cattle and poultry;

+ Map of slaughterhouses and poultry;

+ A map of large food outlets or clusters of food stores that consume large quantities of animal and poultry food;

+ Map of cattle grazing areas of population clusters and farms.

Attribute data:

+ Poultry of each kind (chicken, water bird, bird) are kept in each population cluster, each farm, hatchery, and time of investigation;

+ Number of poultry vaccinated in each population cluster, each farm, hatchery, and injection time.

+ Number of poultry infected with influenza virus in each population cluster, each farm, and breeding farm when epidemic outbreak and time to detect the epidemic.

+ Number of cattle of each type (buffaloes, cows, pigs, and goats) raised in each population cluster, each farm, hatchery, and time of investigation;

+ The number of animals vaccinated in each population cluster, each farm, hatchery, and time of injection.

+ The number of cattle infected with foot-mouth disease in each clustered population, each farm, and breeding farm when epidemic outbreak and the time to detect the epidemic.

+These data are collected over time, used to monitor livestock development, vaccination, and history of avian influenza, foot and mouth disease in each cluster, farm, ranch, hatchery;

+ The trading of cattle and poultry products between population clusters and markets, between localities: People in each population cluster often buy and sell in any market in the region, including the provincial market and other provinces; livestock products, in addition to bringing the market to sell, also exported to other areas;

+ Trading in cattle and poultry breeds among population groups, farms with hatcheries;

+ The relation of trading in animal and poultry feeds among food sellers, feed companies with population clusters, farms, and hatcheries;

+ Sale and purchase of livestock and poultry products between slaughter houses and population clusters and farms;

+ Information on the use of leftovers from food stores for breeding in population clusters, farms, and hatcheries;

+ Location of cattle grazing of population clusters, farms, hatcheries.

\section{Software to Monitor Livestock Situation, Foot-and-Mouth Disease, and Predict the Spread of Influenza}

The client software is the user's tool to log into the system, update information or query, exploit information. The software provides users with the following main functional groups:

The function of updating information and data from the localities in the system helps to update the information in a timely manner, reflecting the situation of breeding and disease in each locality. The updated data in the system are the result of the survey on breeding, the results of vaccination in each wave, the number of poultry flu or the number of cattle with foot-and-mouth disease, the flow of seeds, products, pet food, and other data for the forecasting problem. Local workstations can connect to servers in the Internet, if the server has an address on the network. In cases where the server does not have an address on the network, clients can connect to the server via a public telephone line or use VPN technology. This flexible operating model enables 
the deployment of system applications in all conditions. Data, information is updated by territory, convenient for management work, while reducing the workload of data entry in the storage centre, where to host.

According to Wehrmann and Glavina (2009), one of the differences demonstrating the superiority of using GIS technology is the ability to map the current status of animal husbandry, current status of vaccination, and maps of the current status of influenza, foot and mouth disease (epidemiological map) of the system. Users can choose different criteria according to their needs to build maps, choose different ways of expressing them, such as colouring in absolute value, meaning that each number corresponds to one colour, or fill in the value range. Users can also use dot density on the map to indicate the magnitude of the value to represent instead of colour [1].

Highlights of the system are the ability to predict the spread of avian influenza, foot and mouth disease when the epidemic occurs. The system uses a GIS map to identify areas affected by the spread of the H5N1 virus, or foot-and-mouth disease, in the air from infected subjects. At the same time, combined with the information on trading relations between cattle and poultry products, trading of cattle and poultry breeds, exchanges between population quarters, farms and hatcheries through the market system, slaughterhouses, food stores are stored in the database on the server, the system gives results forecasting objects likely to be infected by the translated epidemic. The probability of being infected is classified as direct infection (level 1), contagious level 2, level 3. According to forecasts, the map colouring system is epidemic to spread the report, set up a synthesis report, make statistics of the number of poultry or cattle in the danger area. Predictors play a very important role in the selection of anti-epidemic tactics such as isolation, remote vaccination, epidemic shedding, and animal husbandry support. The software will explain, on the basis of which a population, farm, hatchery is expected to be infected. The software demonstrates its interpretation in graphic and written form.

\section{Applicable Scope and Possibility}

Targeted monitoring and prediction of avian influenza, foot-and-mouth disease, livestock germs are leaders, officials, and staffs in animal health, from central to local levels, to those who are interested in prevention and control of livestock development.

For commune veterinarians, district veterinary stations, GIS is a tool for updating the database to share livestock survey data, vaccination outcomes, influenza situation, and outbreaks, mouth-to-mouth, trading of breeds, livestock products, trading of animal feeds, and information related to epidemics in their localities. The system is also a tool for them to monitor the situation of poultry production, the situation of avian influenza in their localities and localities, especially localities having exchanges with breeders and producers, poultry products with residents in their localities, the problem of predicting the spread of epidemics when epidemic breaks out.

For leaders of associations, the information system is a tool to store and synthesize information, set up reports, set up status maps. animal husbandry, current status of avian influenza and foot-and-mouth disease, livestock epidemic, progress of vaccination of communes, districts, provinces, and the country. The system provides them with information on the history of livestock development, history of vaccination, history of avian influenza, foot and mouth disease of each commune or country. When the epidemic appears somewhere, the system performs the problem of predicting the spread of epidemic, locating dangerous areas, statistics on the number of poultry are at risk of infection. This is very important information for the prevention and fighting [4]. 
Hardware requirements for the operation of information systems are not complex. At the data management unit, for example, the Animal Health Department, for example, has a data storage server, which has an LAN connection, the Internet, and district health stations having computers and equipment. Internet connection is possible to deploy system applications.

In a nutshell, the information system can be applied to the monitoring of animal husbandry, disease prevention, and control of a district, a province or nationwide. Up to now, the system has been piloted for Vinh Phuc Province, two districts of Ninh Thuan and Hai Hau in Nam Dinh Province, for Quynh Luu district, Nghe An Province and is being applied for the whole province of Quang Ninh Province.

\section{Socio-Economic Efficacy of Application of Information System}

The information system of surveillance and prediction of the spread of avian influenza, foot and mouth disease is a tool for management in veterinary medicine. Its application can't be counted as specific money, but lies in the effectiveness of minimizing the harm caused by the reform of information management methods.

Implementing the information system will help managers to catch the disease quickly and accurately in the area they are in charge. Full and accurate information on the current status of livestock production, distribution maps of densities of livestock and poultry in residential areas provided by the system will help identify locations where poultry raising has too high limits allowed, affect the environment, and be potential for large outbreaks. From that, the management agency should take measures to recommend, orient, and encourage farmers to implement measures to reduce the risk of disease outbreaks, the risk of environmental pollution. Full and accurate information on the current status of animal husbandry helps to prepare appropriate materials, equipment, and human resources for prevention. Having accurate and timely information on where the disease is occurring, on the number of animals and poultry in the affected areas, and on the distribution of infected sites, we will get the instructions to localities in a timely manner, zoning off in the right place, use human resources where necessary, and take measures to prevent the rational and effective.

When it is necessary to incinerate livestock, accurate information on the current status of livestock, the history of animal husbandry in each farm and each village is stored in the database to help estimate the cost of incinerator support and support producers more accurately, contributing to support funds to the right address, the right recipient. Avoiding the situation, when burnt new survey, statistics, led to the passive manager, and the place in the local statistics can generate negative phenomena, statistics, and false reports. Having a map of the distribution of camps, population clusters, and epidemiological results of the epidemic will help to accurately zone the epidemic, avoiding the situation where the need to destroy. The culture is not destroyed, where there is no risk of infection forced to destroy, creating negative reaction from the farmer.

Although the way of infecting H5N1 viruses on human-animal or animal-animal has not yet been identified, the health experts in the world still do not rule out the risk of avian influenza could turn into a flu pandemic, avoiding bird flu will help prevent the emergence of pandemic influenza.

However, the above-mentioned application efficiency is actually achieved only if the management agency has specific regulations on updating the data on livestock and diseases as well as on the factors related to infection and the individuals responsible for updating the information to the system must be self-aware, aware of the importance of objectivity of the information. Thus, the results of aggregating information, mapping the status quo and epidemiological map, the new forecast results have real meaning, effective for prevention and epidemic prevention [5]. 


\section{Conclusion}

Information system to monitor the situation and predict the spread of foot-and-mouth disease is fully functional to meet the requirements of information management on livestock and the fluctuation of foot-and-mouth disease in order to support decision making localization, prevention of outbreaks. With Vietnamese interface, the system is easy to use, without high requirements on hardware, then can deploy applications at different level, especially the provincial level. Currently, the system is being deployed for the whole Quang Ninh province. The information system to monitor the situation and forecast the spread of foot and mouth disease is also the initial basis for development, gradually integrating map layers and data tables. The software module monitors the situation and predicts the spread of other dangerous diseases.

\section{References}

Gleeson, L. (2003). A review of the status of foot and mouth disease in South-East Asia and approaches to control and eradication. Revue scientifique et technique, 21, 465-475. 10.20506/rst.21.3.1346.

Huisman, O., \& Rolf, A. D. (2011). Principles of geographic information systems. An introductory textbook. The International Institute for Geo-Information Science and Earth Observation (ITC).

Pendell, D. L., Leatherman, J., Schroeder, T. C., \& Alward, G. S. (2007). The economic impacts of a foot-and-mouth disease outbreak: A regional analysis. Western Agricultural Economics Association Annual Meeting, Portland, OR.

Truong, D. B., Goutard, F. L., Bertagnoli, S., Delabouglise, A., Grosbois, V., \& Peyre, M. (2018). Benefit-cost analysis of foot-and-mouth disease vaccination at the farm-level in South Vietnam. Frontiers in Veterinary Science, 5, $10.3389 /$ fvets.2018.00026.

Wehrmann, B., \& Glavina, J. (2009). Geographic information systems (GIS). SELLER Sector Network Sector Project Land Management. 